\title{
Downregulated long non-coding RNA DREH promotes cell proliferation in hepatitis B virus-associated hepatocellular carcinoma
}

\author{
DONG LV ${ }^{1}$, YUAN WANG ${ }^{2}$, YING ZHANG $^{1}$, PEILIN CUI $^{1}$ and YOUQING XU ${ }^{1}$ \\ ${ }^{1}$ Gastroenterology Department, Beijing Tiantan Hospital, Capital Medical University, Beijing 100050; \\ ${ }^{2}$ Third Department of General Surgery, Peking University People's Hospital, Beijing 100044, P.R. China
}

Received March 27, 2016; Accepted March 23, 2017

DOI: 10.3892/ol.2017.6436

\begin{abstract}
The hepatitis B virus X ( $\mathrm{HBx})$ protein has been characterized as an oncogene involved in epigenetic modifications during hepatocarcinogenesis; however, the underlying mechanisms are not entirely clear. Long non-coding RNAs (lncRNAs), a type of epigenetic regulator molecules, have also been demonstrated to serve crucial roles in carcinogenesis, including hepatocellular carcinoma (HCC). In the present study, a human IncRNA DREH was identified, which inhibits cell proliferation in vitro and in vivo, and acts as a tumor suppressor in $\mathrm{HBx}$-mediated hepatocarcinogenesis. The study revealed that the expression of DREH was frequently downregulated in hepatitis B virus (HBV)-associated HCC tissues in comparison with adjacent non-cancerous hepatic tissues, and was inversely correlated with $\mathrm{HBx}$ mRNA expression in $\mathrm{HBV}$-associated HCC. In addition, the levels of DREH were inversely correlated with hepatitis B surface antigen and tumor size in HCC tissues. The forced expression of $\mathrm{HBx}$ in liver cell lines resulted in a significant decrease in the expression of DREH. Furthermore, suppression of DREH expression promotes the proliferation of HCC cells in vitro and in vivo. In conclusion, the present findings support the role of HBx-downregulated lncRNA DREH in tumor suppression in HBV-associated HCC patients. This contributes to a better understanding of epigenetic aberration of
\end{abstract}

Correspondence to: Dr Dong Lv, Gastroenterology Department, Beijing Tiantan Hospital, Capital Medical University, 6 Tiantan Xi Li, Dongcheng, Beijing 100050, P.R. China

E-mail: tiantanlvdong@sina.com

Abbreviations: $\mathrm{HBV}$, hepatitis B virus; $\mathrm{HBx}$, hepatitis B virus $\mathrm{X}$; HCC, hepatocellular carcinoma; lncRNA, long non-coding RNA; HBsAg, hepatitis B surface antigen; AFP, $\alpha$-fetoprotein; ALT, alanine aminotransferase; RT-qPCR, reverse transcription-quantitative polymerase chain reaction; siRNA, small interfering RNA; CCK-8, Cell Counting Kit-8

Key words: hepatocellular carcinoma, hepatitis B virus $\mathrm{X}$ protein, long non-coding RNA, cell proliferation, DREH deregulated lncRNAs by HBx and the potential development of IncRNA-based targeted approaches for the treatment of HBV-associated HCC.

\section{Introduction}

As one of the most common cancer types in the world, hepatocellular carcinoma (HCC) has an extremely high morbidity and mortality rate, particularly in Asia and Africa (1). Overall, 50 to $55 \%$ of $\mathrm{HCC}$ cases are attributable to persistent hepatitis $\mathrm{B}$ virus (HBV) infections, which may result in end-stage liver disease, including liver cirrhosis and HCC (2). As the smallest open reading frame of the $\mathrm{HBV}$ genome, $\mathrm{HBX}$ encodes the hepatitis $\mathrm{B}$ virus $\mathrm{X}(\mathrm{HBx})$ protein which has been implicated in HBV-associated HCC pathogenesis, acting as a weak oncogene or a cofactor in hepatocarcinogenesis (3-5). However, the molecular mechanisms underlying HBx protein-mediated tumorigenesis are not entirely clear. Previous studies have demonstrated that genetic alterations alone do not account for the complexity of $\mathrm{HBx}$-induced hepatocarcinogenesis, but that epigenetic changes, including DNA methylation (6), histone modifications (7) and non-coding RNA expression $(6,8)$, are also involved in this process.

Long non-coding RNAs (lncRNAs) are a type of non-coding RNAs which are longer than 200 nucleotide transcripts and have little or no protein-coding capacity $(9,10)$. Previous studies have demonstrated that lncRNAs are involved in diverse biological functions and pathological processes $(10,11)$, and that altered 1 ncRNA levels may result in aberrant gene expression through a variety of mechanisms, including transcription, post-transcriptional processing (12), chromatin modification, genomic imprinting and the regulation of protein function (13). Increasing evidence demonstrates that altered expression levels of lncRNAs contribute to a wide range of cancer types, including breast, lung, prostate and liver cancer (14-17). Therefore, IncRNAs may potentially be used as diagnostic markers or therapeutic targets for cancer in the clinic.

Using lncRNA microarrays and gene sequencing technology, a large number of lncRNAs have been observed to be aberrantly expressed in HCC tissues and involved in hepatocarcinogenesis. These include highly upregulated in liver 
cancer (HULC), high expression in HCC (HEIH), activated by TGF- $\beta$ (ATB) and HOX transcript antisense RNA (HOTAIR), which serve a role in diverse biological processes including cell proliferation, apoptosis and metastasis (17-20). Several IncRNAs have been identified to be associated with the HBx protein $(17,21)$. Huang et al $(22)$ examined the lncRNA expression profiles in the livers of $\mathrm{HBx}$ transgenic and wild-type mice, and observed that certain IncRNAs are dysregulated and associated with HBx in HBx transgenic mice. These authors further investigated the biological function of the lncRNA Dreh, which may be downregulated by HBx protein, in mice. It was observed to inhibit HCC growth and metastasis, acting as a tumor suppressor in the development of HBV-HCC. The same authors also identified a human ortholog of Dreh, which was termed DREH, and observed that its expression level was frequently downregulated in HBV-associated HCC tissues. This decrement was significantly correlated with poor survival in HCC patients. However, the specific role of IncRNA DREH in HCC remains largely unknown.

In the present study, the expression levels of IncRNA DREH in 30 pairs of human HBV-positive HCC tissues and 30 pairs of HBV-negative HCC tissues and their pair-matched normal liver tissues were assessed. The results revealed that the expression level of DREH was significantly downregulated in HBV-HCC tissues compared with their adjacent non-cancerous hepatic tissues, and was inversely correlated with $\mathrm{HBx}$ mRNA expression in HBV-associated HCCs. Further investigation of the biological function of DREH in vivo and in vitro revealed that inhibition of DREH promotes cell proliferation in HBx-induced hepatocarcinogenesis. Together, these results suggest that DREH exerts an impact as a potential tumor repressor gene and may provide new insight into the role of HBx-associated lncRNAs in the development of HCC.

\section{Materials and methods}

Animal and patient samples. The four-week-old male $\mathrm{BALB} / \mathrm{c}$ nude mice used in this study were purchased from the Experimental Animal Center of the Chinese Academy of Medical Sciences (Beijing, China). All mice were bred and maintained in a pathogen-free facility and were used in accordance with the institutional guidelines for animal care. The animal studies were approved by the Institutional Animal Care and Use Committee of the Capital Medical University, Beijing, China.

The $30 \mathrm{HBV}$-associated HCC tissues and $30 \mathrm{HBV}$-negative HCC tissues and corresponding adjacent non-cancerous liver tissues used in this study were obtained with informed consent from patients who underwent radical resection in the Peking University People's Hospital (Beijing, China). Studies using human tissues were reviewed and approved by the Committees for Ethical Review of Research Involving Human Subjects of the Capital Medical University. The clinicopathological characteristics of the 60 patients are summarized in Table I.

Construction of vectors. To construct $\mathrm{HBx}$-expressing vectors, complementary DNA encoding HBx was PCR-amplified and sub-cloned into the pcDNA3.1 vector (Invitrogen; Thermo Fisher Scientific, Inc., Waltham, MA, USA). All vectors were constructed according to standard methods and verified by
Table I. Clinicopathological characteristics of $60 \mathrm{HCC}$ patients.

\begin{tabular}{lcc}
\hline Characteristic & Number $(\mathrm{n}=60)$ & Percentage \\
\hline Age (years) & & \\
$\quad \leq 55$ & 21 & 65.00 \\
$>55$ & & 35.00 \\
Sex & 51 & \\
$\quad$ Male & 9 & 85.00 \\
Female & & 15.00 \\
Tumor differentiation & 21 & \\
I-II & 39 & 35.00 \\
III-IV & & 65.00 \\
TNM stage & 25 & 41.67 \\
I & 35 & 58.33 \\
II-III & & \\
Tumor size (cm) & 31 & 51.67 \\
$\leq 5$ & 29 & 48.33 \\
$>5$ & &
\end{tabular}

Tumor number

Single

52

86.67

Multiple

8

13.33

$\operatorname{AFP}(\mu \mathrm{g} / \mathrm{l})$

$\leq 20$

12

20.00

$>20$

80.00

Encapsulation

Absent

48.33

Complete

51.67

Microvascular invasion

Absent

Present

29

85.00

15.00

Macrovascular invasion

Absent

51

9

90.00

Present

54

10.00

Liver cirrhosis

Absent

Present

HBs antigen

Negative

30

50.00

Positive

30

50.00

$\mathrm{HBe}$ antigen

Negative

40

66.67

Positive

20

33.33

ALT (U/l)

$\leq 40$

26

43.33

$>40$

34

56.67

HCC, hepatocellular carcinoma; TNM, tumor-node-metastasis; AFP, $\alpha$-fetoprotein; HBs antigen, hepatitis B surface antigen; HBe antigen, hepatitis B envelope antigen; ALT, alanine aminotransferase.

sequencing. The polymerase chain reaction (PCR) primers used are presented in Table II. 
Table II. Sequences of primers and siRNAs used in study.

\begin{tabular}{|c|c|c|}
\hline Name & & Sequences \\
\hline \multicolumn{3}{|l|}{ qPCR primers } \\
\hline \multirow[t]{2}{*}{$\mathrm{HBx}$} & Sense & 5'-СССТTCTTCATCTACCGTTCC-3' \\
\hline & Anti-sense & 5'-CGTTGACATTGCTGCGAGT-3' \\
\hline \multirow[t]{2}{*}{$\beta$-actin } & Sense & 5'-TGTGTTGGCGTACAGGTCTTTG \\
\hline & Anti-sense & 5'-GGGAAATCGTGCGTGACATTAAG \\
\hline \multirow[t]{2}{*}{ DREH } & Sense & 5'-CATTTGGCGGGACTACTTATT-3' \\
\hline & Anti-sense & 5'-TTCAATCTGGCTTTGTTCGTT-3' \\
\hline \multicolumn{3}{|c|}{ Primers for vector construction } \\
\hline \multirow[t]{2}{*}{ DREH clone } & Sense & 5'-GGGGTACCCCATGGCTGCTAGGGTGTG-3' \\
\hline & Anti-sense & 5'-CGGGATCCCGTCAGGCAGAGGTGAAAAAG-3' \\
\hline \multicolumn{3}{|c|}{ siRNA sequences } \\
\hline \multirow[t]{2}{*}{ DREH siRNA } & Sense & 5'-UCAUUUGGCGGGACUACUUTT-3' \\
\hline & Anti-sense & 5'-AAGUAGUCCCGCCAAAUGATT-3' \\
\hline \multirow[t]{2}{*}{ siRNA NC } & Sense & 5'-UUCUCCGAACGUGUCACGUTT-3' \\
\hline & Anti-sense & 5'-ACGUGACACGUUCGGAGAATT-3' \\
\hline \multirow[t]{2}{*}{ HBx siRNA } & Sense & 5'-CCCACCAAAUAUUGCCCAATT-3' \\
\hline & Anti-sense & 5'-UUGGGCAAUAUUUGGUGGGTT-3' \\
\hline
\end{tabular}

siRNA, small interfering RNA; qPCR, quantitative polymerase chain reaction; HBx, hepatitis B virus X; NC, negative control.

Cell culture and transfection. The liver cell lines HepG2, HepG2.2.15, Hep3B, Huh-7 and SMMC-7721 were obtained from the American Type Culture Collection (Manassas, VA, USA). The cells were grown in Dulbecco's modified Eagle's medium (Gibco; Thermo Fisher Scientific, Inc.) with $10 \%$ fetal bovine serum (Gibco) and were maintained in a humidified $37^{\circ} \mathrm{C}$ incubator with an atmosphere of $5 \% \mathrm{CO}_{2}$. The different plasmids and small interfering RNA (siRNA) sequences were transfected into cells using a Lipofectamine ${ }^{\circledR} 3000$ kit (Invitrogen) according to the manufacturer's protocol. The siRNAs were synthesized by GenePharma (Shanghai, China). The siRNA sequences are provided in Table II.

Reverse transcription and quantitative PCR (RT-qPCR). Total RNA was extracted using TRIzol reagent (Invitrogen). First-strand cDNA was generated using the Reverse Transcription system kit (Stratagene, La Jolla, CA, USA). Random primers (6mer; Takara Bio, Inc., Otsu, Japan) were used for RT-PCR for lncRNAs. Real-time PCR was performed using a standard SYBR-Green PCR kit protocol on a StepOne Plus system (Applied Biosystems, Thermo Fisher Scientific, Inc.). $\beta$-actin was employed as an endogenous control to normalize for the amount of total mRNA in each sample. The qPCR reactions were performed in triplicate, including no-template controls. The relative RNA expression was calculated using the comparative $\mathrm{Cq}$ method. The primer sequences are presented in Table II.

Cell Counting Kit-8 (CCK-8) assay. HepG2 or Huh-7 cells $\left(2 \times 10^{3}\right.$ cells/well) transfected with DREH siRNA or negative control were dispensed in 100- $\mu$ l aliquots into 96-well plates. At the indicated time points, CCK-8 (Dojindo Molecular Technologies, Inc., Kumamoto, Japan) was added to the cells for $2 \mathrm{~h}$ and then the optical density was read using a microplate reader (Bio-Rad Laboratories, Inc., Hercules, CA, USA). All of the experiments were performed in triplicate.

Colony formation assay. For colony formation assay, cells were seeded at a density of 100 cells per well in a 12 -well culture plate and cultured for 2 weeks, then cells were washed twice with phosphate-buffered saline (PBS), fixed with methanol, and the colonies were stained with $1 \%$ crystal violet and counted.

In vivo assay for tumor growth. Lentivirus-based short hairpin RNA (shRNA) constructs (GenePharma) were used to stably knock down DREH gene expression according to the manufacturer's protocol. HepG2 cells were stably transduced with DREH shRNA lentivirus. Cells transfected with DREH shRNA or control shRNA $\left(1.0 \times 10^{7}\right)$ were suspended in $100 \mu \mathrm{l}$ PBS and implanted subcutaneously into the bilateral armpit of BALB/c nude mice (five in each group). The tumors were measured every three days after implantation, and the volume of each tumor was calculated as: Length $\mathrm{x}$ width ${ }^{2} \mathrm{x} 0.4$. All mice were sacrificed four weeks later.

Statistical analysis. The expression of DREH in HCC patients was compared using the paired samples t-test. The association between DREH and HBx mRNA expression was analyzed by Pearson's correlation. The correlations between DREH and clinicopathological characteristics in the $60 \mathrm{HCC}$ patients were analyzed by the $\chi^{2}$ test or Fisher's exact probability test. Others comparisons were determined by Student's t-test. All P-values were two-sided and obtained using the SPSS 18.0 software package (SPSS, Inc., Chicago, IL, USA). $\mathrm{P}<0.05$ was considered to indicate a statistically significant difference. 


\section{Results}

LncRNA DREH is significantly downregulated in $H B V$-associated HCC tissues. To confirm the role of IncRNA DREH in HCC, DREH expression levels were first examined in 30 pairs of human HBV-associated HCC tissues and 30 pairs of HBV-negative HCC tissues and their pair-matched normal liver tissues by qPCR. The results revealed that the expression levels of DREH were significantly downregulated in HBV-HCC tissues in comparison with adjacent non-cancerous hepatic tissues from the same patient $(\mathrm{P}<0.0001$, paired samples $\mathrm{t}$-test); however, no significant difference was observed in the expression levels between the HBV-negative HCC tissues and the adjacent non-cancerous hepatic tissues. In addition, the expression of DREH was significantly higher in HBV-negative HCC tissues compared with HBV-positive HCC tissues (Fig. 1A).

DREH expression was further compared with clinicopathological characteristics in these $60 \mathrm{HCC}$ patients, and statistical analysis revealed that lower DREH expression levels in HCC tissues were significantly positively correlated with tumor size $\left(\chi^{2}=5.406, P=0.020\right.$, Table III $)$ and hepatitis B surface antigen (HBsAg) $\left(\chi^{2}=4.267, \mathrm{P}=0.039\right.$, Table III). However, no direct correlation was identified between the expression of lncRNA DREH and other clinical characteristics, including age, sex, tumor differentiation, tumor-node-metastasis stage, tumor number, $\alpha$-fetoprotein (AFP), encapsulation, microvascular invasion, macrovascular invasion, liver cirrhosis, hepatitis B envelope antigen and alanine aminotransferase (ALT) (Table III). These results indicate that DREH may be involved in HCC tumor growth and potentially associated with HBV infection.

DREH and HBX mRNA levels are inversely correlated in human HBV-associated HCC tissues. Next, it was assessed whether decreased DREH expression was correlated with the levels of HBx expression in human HBV-associated HCC tissues. The expression levels of $\mathrm{HBx}$ were further analyzed in the aforementioned $30 \mathrm{HCC}$ tissues. A statistically significant inverse correlation was observed between DREH and $\mathrm{HBx}$ mRNA ( $\mathrm{n}=30, \mathrm{r}=-0.531, \mathrm{P}=0.0033$, Pearson's correlation; Fig. 1B). These data reveal the potential reciprocal regulation of DREH expression induced by HBx in human HCCs, and suggest that DREH may be involved in HCC pathogenesis as a tumor suppressor subsequent to HBx overexpression in chronic hepatitis B patients.

DREH is significantly downregulated in human HCC cell lines expressing $H B x$. To investigate the correlation between $\mathrm{HBx}$ and DREH expression, it was first determined whether DREH was differentially expressed in human HCC cells. The expression levels of DREH were assessed by RT-qPCR. The results revealed that the expression of DREH was markedly lower in HepG2.2.15 (a derivative of the human hepatoma cell line HepG2 that has been stably transformed with a head-to-tail dimer of HBV DNA) and Hep3B (a cell line containing the integrated hepatitis B viral genome) cell lines compared with HepG2, Huh-7 and SMMC-7721 cells, which do not express HBx (Fig. 2A).

Enforced HBx expression downregulates DREH in human $H C C$ cells. In order to verify whether this downregulation was
Table III. Correlation between IncRNA DREH expression and clinicopathological characteristics in $60 \mathrm{HCC}$ patients.

\begin{tabular}{|c|c|c|c|}
\hline \multirow[b]{2}{*}{ Characteristic } & \multicolumn{2}{|c|}{$\begin{array}{l}\text { LncRNA DREH } \\
\text { expression }\end{array}$} & \multirow[b]{2}{*}{ P-value } \\
\hline & $\begin{array}{c}\text { Low } \\
(\mathrm{n}=30)\end{array}$ & $\begin{array}{l}\text { High } \\
(\mathrm{n}=30)\end{array}$ & \\
\hline Age (years) & & & 0.787 \\
\hline$\leq 55$ & 20 & 19 & \\
\hline$>55$ & 10 & 11 & \\
\hline Sex & & & 0.278 \\
\hline Male & 24 & 27 & \\
\hline Female & 6 & 3 & \\
\hline Tumor differentiation & & & 0.787 \\
\hline I-II & 10 & 11 & \\
\hline III-IV & 20 & 19 & \\
\hline TNM stage & & & 0.190 \\
\hline I & 10 & 15 & \\
\hline II-III & 20 & 15 & \\
\hline Tumor size $(\mathrm{cm})$ & & & $0.020^{\mathrm{a}}$ \\
\hline$\leq 5$ & 20 & 11 & \\
\hline$>5$ & 10 & 19 & \\
\hline Tumor number & & & $0.254^{\mathrm{b}}$ \\
\hline Single & 24 & 28 & \\
\hline Multiple & 6 & 2 & \\
\hline $\operatorname{AFP}(\mu \mathrm{g} / \mathrm{l})$ & & & $0.333^{\mathrm{b}}$ \\
\hline$\leq 20$ & 4 & 8 & \\
\hline$>20$ & 26 & 22 & \\
\hline Encapsulation & & & 0.196 \\
\hline Absent & 12 & 17 & \\
\hline Complete & 18 & 13 & \\
\hline Microvascular invasion & & & $1.000^{\mathrm{b}}$ \\
\hline Absent & 25 & 26 & \\
\hline Present & 5 & 4 & \\
\hline Macrovascular invasion & & & $0.671^{b}$ \\
\hline Absent & 26 & 28 & \\
\hline Present & 4 & 2 & \\
\hline Liver cirrhosis & & & 0.754 \\
\hline Absent & 6 & 7 & \\
\hline Present & 24 & 23 & \\
\hline HBs antigen & & & $0.039^{\mathrm{a}}$ \\
\hline Negative & 11 & 19 & \\
\hline Positive & 19 & 11 & \\
\hline HBe antigen & & & 0.273 \\
\hline Negative & 18 & 22 & \\
\hline Positive & 12 & 8 & \\
\hline $\operatorname{ALT}(\mathrm{U} / \mathrm{l})$ & & & 0.297 \\
\hline$\leq 40$ & 11 & 15 & \\
\hline$>40$ & 19 & 15 & \\
\hline
\end{tabular}

${ }^{\mathrm{a}} \mathrm{P}<0.05$; ${ }^{\mathrm{b}}$ Fisher's exact test; $\chi^{2}$ tests for all other analyses. The median expression level of lncRNA DREH was used as the cutoff. Patients with HC C were divided into an lncRNA DREH 'low' group (whose expression was lower than the median) and 'high' group (whose expression was higher than the median). LncRNA, long non-coding RNA; HCC, hepatocellular carcinoma; AFP, $\alpha$-fetoprotein; HBs antigen, hepatitis B surface antigen; HBe antigen, hepatitis B envelope antigen; ALT, alanine aminotransferase. 

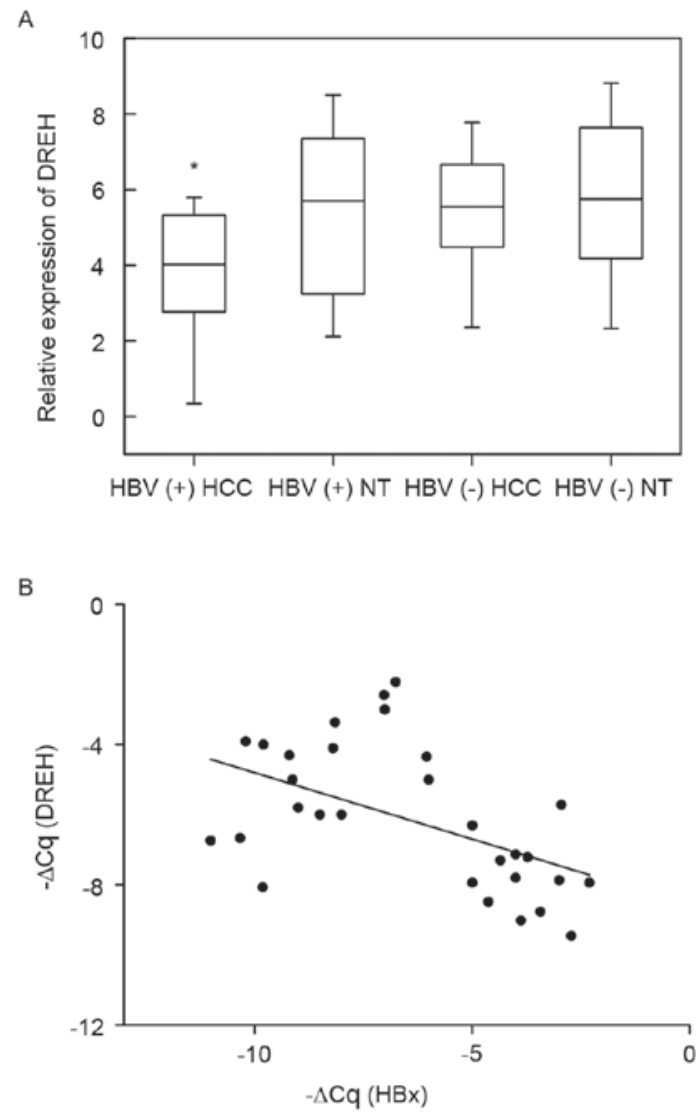

Figure 1. LncRNA DREH is significantly downregulated in HBV-associated HCC tissues. (A) LncRNA DREH expression in HCC tissues vs. paired adjacent non-cancerous hepatic tissues by qPCR (from 30 pairs of HBV-associated HCC patients and 30 pairs of HBV-negative HCC patients). Statistical differences between HBV-HCC tissues and paired adjacent non-cancerous hepatic tissues were analyzed with the paired samples t-test ( $\mathrm{P}<0.0001)$. (B) LncRNA DREH and HBx mRNA expression levels were inversely correlated in 30 $\mathrm{HBV}$-associated HCC samples. DREH and HBx expression levels in these samples were measured by qPCR, and respective $\Delta \mathrm{Cq}$ values normalized to $\beta$-actin were subjected to a Pearson correlation analysis $(n=30, r=-0.531$, $\mathrm{P}=0.0033$, Pearson's correlation). LncRNA, long non-coding RNA; HBV, hepatitis B virus; HCC, hepatocellular carcinoma; qPCR, quantitative polymerase chain reaction; HBx, hepatitis B virus X; NT, normal tissues.

correlated with HBx expression, HepG2 and Huh-7 cells were transiently transfected with $\mathrm{HBx}$ expression vector pc-HBx and control vector pcDNA3.1. The levels of DREH were measured $72 \mathrm{~h}$ after transient transfection. The mRNA expression of HBx following infection is shown in the left panel of Fig. 2B. The results reveal that DREH was downregulated in pc-HBx-transfected cells in comparison with the pcDNA3.1 control groups (Fig. 2B).

Conversely, HBx expression was also repressed by siRNA; the knockdown efficacy of HBx siRNA is shown in the left panel of Fig. 2C. The inhibition of HBx by siRNA was observed to increase DREH expression in HepG2.2.15 and Hep3B cells which express HBx (Fig. 2C).

Inhibition of DREH promotes cell proliferation of HCC cells in vitro. The frequent downregulation of IncRNA DREH by HBx and the inverse correlation between DREH expression and tumor size in HBV-HCC patients implies that DREH may have a role in cell proliferation in HBV-associated hepatocarcinogenesis. To prove this, the effects of reduced expression of
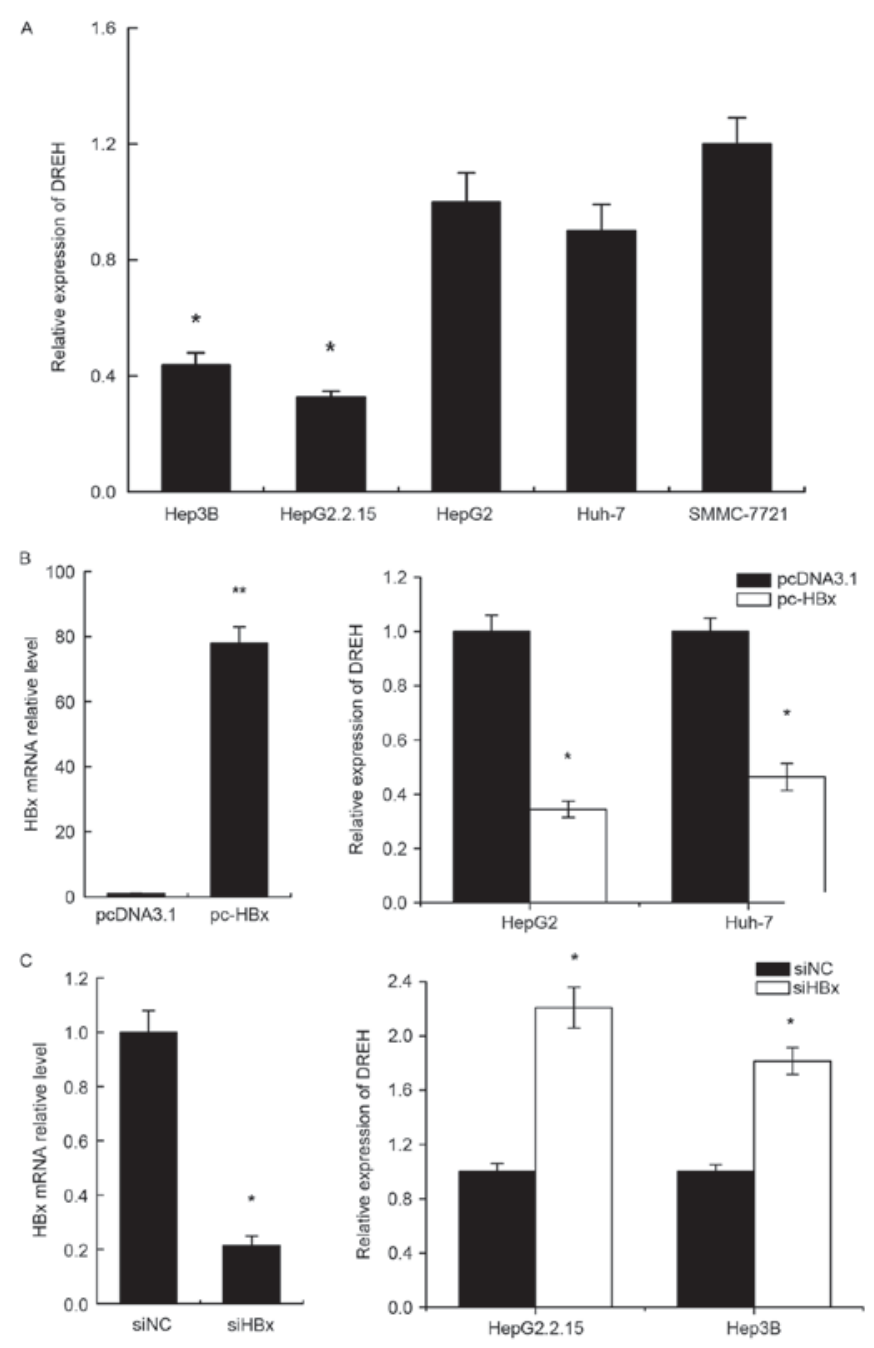

Figure 2. DREH is significantly downregulated in human HCC cell lines expressing HBx. (A) Relative expression of DREH in human liver cell lines Hep3B, HepG2.2.15, HepG2, Huh-7 and SMMC-7721 cells. The expression of DREH was normalized to $\beta$-actin with respect to HepG2 cells. Data are shown as the means \pm standard deviation from triplicate experiments. ${ }^{*} \mathrm{P}<0.05$ vs. HepG2 group. (B) HBx mRNA (left panel) and lncRNA DREH (right panel) expression levels following transfection of pc-HBx or control pcDNA3.1 plasmid in HepG2 and Huh-7 cells. Data are shown as the means \pm standard deviation from at least three independent experiments. ${ }^{*} \mathrm{P}<0.05 ;{ }^{* *} \mathrm{P}<0.01$ vs. respective pcDNA3.1 groups. (C) HBx mRNA (left panel) and DREH (right panel) expression levels following transfection of HBx-specific siRNA (siHBx) or control siRNA (siNC) in Hep3B and HepG2.2.15 cells. Data are shown as the means \pm standard deviation from at least three independent experiments. ${ }^{*} \mathrm{P}<0.05$ vs. respective siNC groups. $\mathrm{HBx}$, hepatitis B virus $\mathrm{X}$; NC, negative control.

DREH on cell proliferation were investigated in two HCC cell lines. DREH expression was repressed by RNA interference, and the relative expression levels of DREH following infection of DREH siRNA or control siRNA are shown in the left panel of Fig. 3A and B. Cell-Counting Kit-8 assays demonstrated that suppression of cellular DREH enhanced the cell proliferation index compared with the control siRNA group in HepG2 and Huh-7 cells. The negative control siRNAs did not affect the cell proliferation index compared with the mock cells with no treatment (Fig. 3A and B).

Further colony formation assays also revealed that downregulation of DREH significantly enhanced the colony formation ability in HepG2 and Huh-7 cells compared with the 

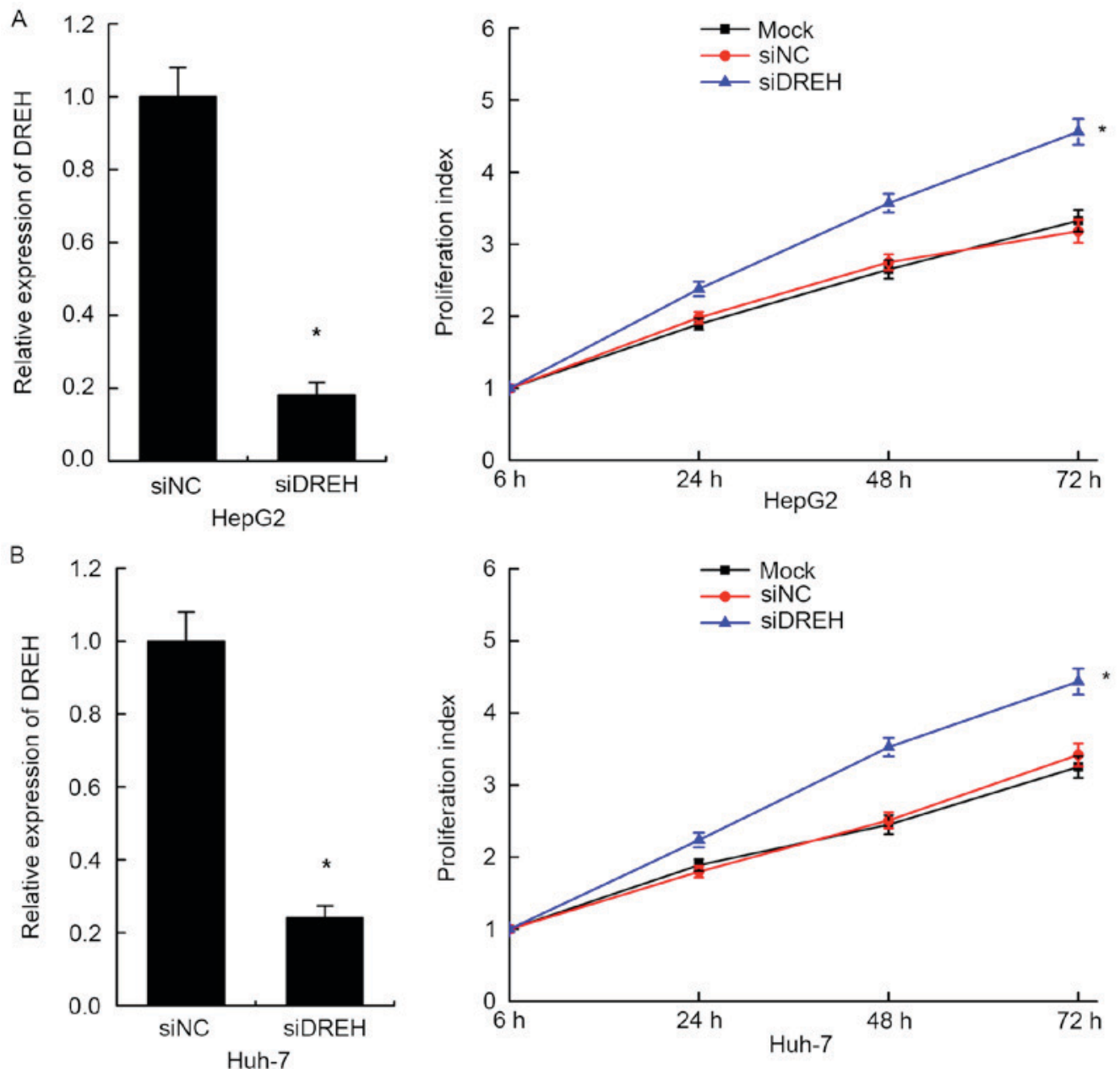

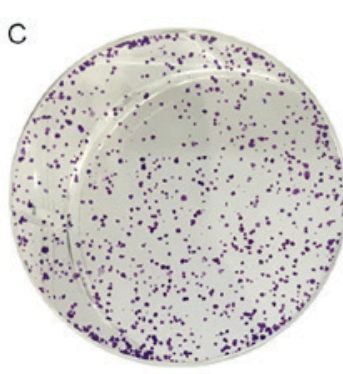

siNC

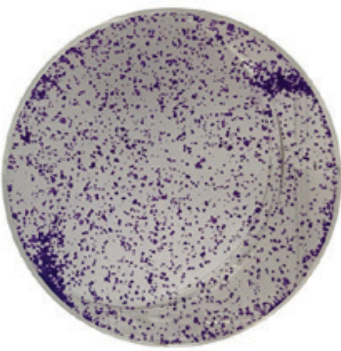

SIDREH

HepG2

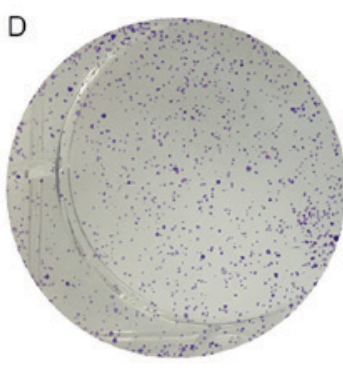

siNC

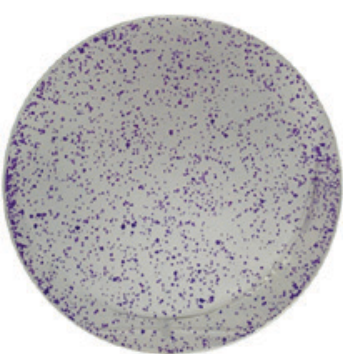

siDREH
Huh-7
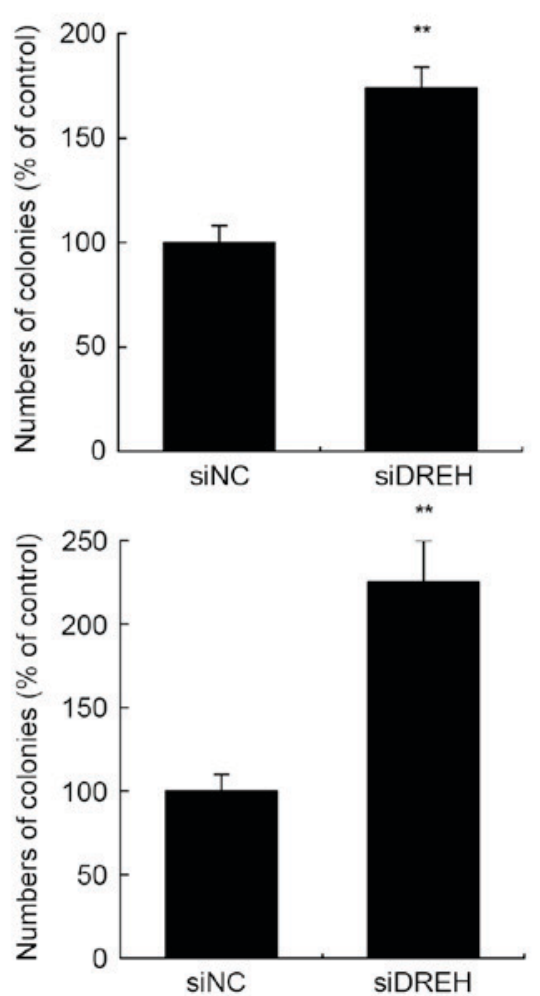

Figure 3. Inhibition of DREH promotes cell proliferation of HCC in vitro. (A) and (B) HepG2 and Huh-7 cells were infected with siRNA against DREH (siDREH) or respective control siRNA (siNC) and control cells without treatment (mock), and the cell proliferation activity was assessed by Cell Counting Kit-8 assay. Following transfection, cells were seeded in 96-well plates, and optical density at $450 \mathrm{~nm}$ was assessed 6, 24, 48 and 72 h after cells were adherent. (C) and (D) Colony formation assays were performed on HepG2 and Huh-7 cells following transfection of siRNA against DREH (siDREH) or respective control siRNA (siNC) Cells were stained with $1 \%$ crystal violet and counted. Data are shown as the means \pm standard deviation based on at least three independent experiments. ${ }^{*} \mathrm{P}<0.05 ;{ }^{* *} \mathrm{P}<0.01$ vs. siNC group. 


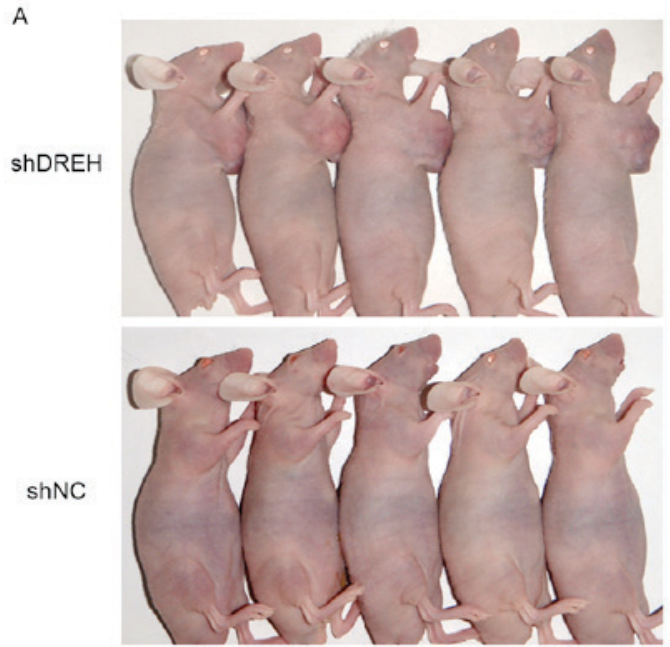

C

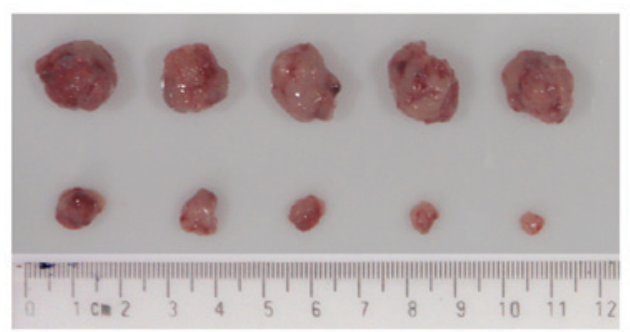

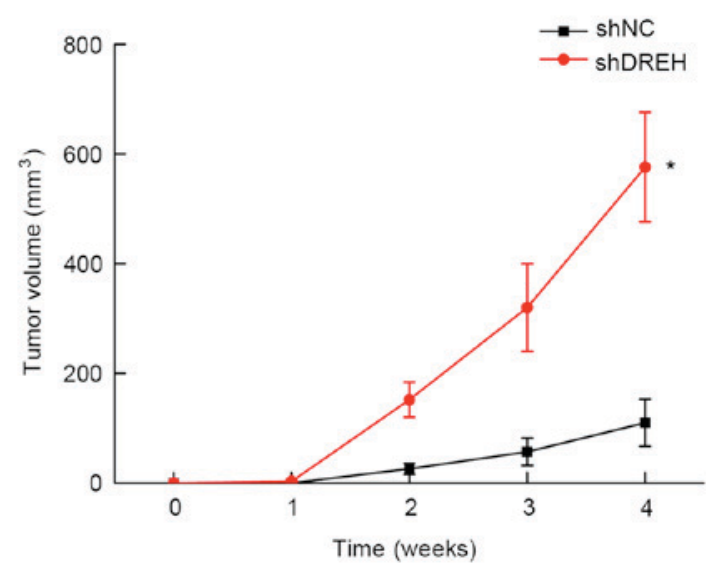

D

ShDREH

shNC

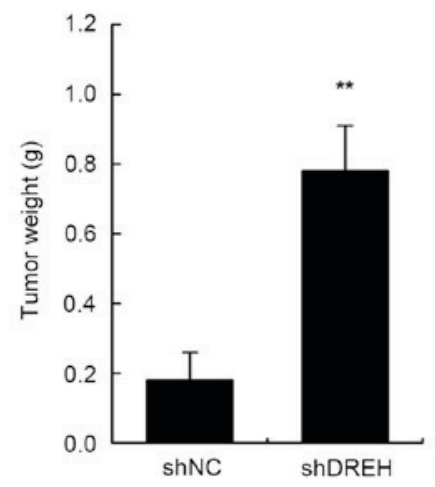

Figure 4. Inhibition of DREH promotes tumor growth in vivo. (A) Images of tumors that developed in xenograft-transplanted nude mice four weeks after cell injection [HepG2 with shRNA against DREH (shDREH) or control shRNA (shNC)]. (B) Tumor volume in the two groups of nude mice following ectopic subcutaneous implantation of HepG2 cells transfected with shDREH or shNC. (C) Images of tumor xenografts 4 weeks after ectopic subcutaneous implantation in nude mice. (D) Effect of shDREH or shNC on hepatocellular carcinoma tumor growth was described by tumor weight in the two groups. Data are shown as the means \pm standard deviation. ${ }^{*} \mathrm{P}<0.05 ;{ }^{* *} \mathrm{P}<0.01$ vs. shRNA NC group.

control cells, consistent with the above results (Fig. 3C and D). Thus, these results suggest that DREH may serve a key role in HBx-induced hepatocellular proliferation.

Inhibition of DREH promotes tumor growth in vivo. To determine the effects of DREH on tumorigenesis in vivo, DREH-downregulated or control cells (HepG2 cells stably transfected with either shRNA-DREH or control shRNA) were subcutaneously injected into nude mice for xenoplantation. Mice injected with cells transfected with shRNA-DREH demonstrated significantly increased tumor growth compared with those injected with cells transfected with control shRNA (Fig. 4A and C).

As assessed by measurements of tumor volume, tumor weight and tumor weight/body weight ratio, the inhibition of DREH expression significantly promoted overall tumor growth 4 weeks after ectopic subcutaneous implantation in nude mice (Fig. 4B and D). These results further indicated that DREH was involved in the biological function of cell proliferation in HBV-associated HCC.

\section{Discussion}

HCC is a leading cause of cancer-associated mortality worldwide (23). Current guidelines recommend different therapeutic measures for the treatment of HCC patients with different stages, including surgery, chemotherapy, radiation therapy, and sorafenib and transarterial chemoembolization $(24,25)$. Despite several recent advances and technical refinements, the long-term survival outcome of patients remains unsatisfactory (26). Therefore, it is necessary to thoroughly investigate the pathogenetic mechanism of HCC and develop new targeted treatments. The majority of recent investigations into cancer etiology have identified that epigenetics serves a critical role in cancer $(27,28)$. Alterations in epigenetic modifications regulate all DNA-based processes, including transcription, DNA repair and replication, and are considered to be early events in tumorigenesis. There are also potential targets for therapeutic intervention using epigenetic drugs $(29,30)$.

LncRNAs are a type of epigenetic regulator and are becoming one of the hot topics in genome research. Previous studies have revealed various functions and molecular mechanisms of these enigmatic molecules in biological processes of human health and diseases $(31,32)$. With the development of high-throughput detection technologies including lncRNA microarray, RNA sequencing and the recent application of next-generation sequencing, thousands of lncRNAs have been observed to be aberrantly expressed and associated with various cancer types (33). The HBx protein has been reported to promote malignant transformation by epigenetic modifications and genetic regulation during hepatocarcinogenesis $(34,35)$. HBx also alters the expression profiles of 
lncRNAs, and these cancer-associated lncRNAs may serve key roles in gene regulation and thus affect various aspects of cellular homeostasis $(21,22)$.

In this study, a human lncRNA DREH was identified, which was downregulated by HBx protein. The suppression of DREH expression promotes the proliferation of HCC cells in vitro and in vivo, acting as a tumor suppressor in $\mathrm{HBx}$-mediated hepatocarcinogenesis. The expression levels of DREH were examined in 30 pairs of human HBV-positive HCC tissues and 30 pairs of HBV-negative HCC tissues and their pair-matched normal liver tissues. The results revealed that the expression of DREH was frequently downregulated in HBV-associated HCC tissues compared with their adjacent non-cancerous hepatic tissues and was inversely correlated with HBx mRNA expression in $\mathrm{HBV}$-associated HCCs. Clinical correlation analysis demonstrated that the levels of DREH were inversely correlated with HBsAg and tumor size in HCC tissues.

In summary, these findings suggest that lncRNA DREH exerts an impact as a potential tumor repressor gene in the development of human HBV-associated HCC. The modulation of cell proliferation by DREH may be used as a potential target for the prevention and treatment of HBV-associated HCC.

\section{References}

1. El-Serag HB and Rudolph KL: Hepatocellular carcinoma: Epidemiology and molecularcarcinogenesis. Gastroenterology 132: 2557-2576, 2007.

2. Bosch FX, Ribes J, Diaz M and Cléries R: Primary liver cancer: Worldwide incidence and trends. Gastroenterology 127 (5 Suppl 1): 5-16, 2004.

3. Koike K: Hepatitis B virus HBx gene and hepatocarcinogenesis. Intervirology 38: 134-142, 1995.

4. Huang P, Zhuang B, Zhang H, Yan H, Xiao Z, Li W, Zhang J, Tang Q, Hu K, Koeffler HP, et al: Hepatitis B virus X protein $(\mathrm{HBx})$ is responsible for resistance to targeted therapies in hepatocellular carcinoma: Ex vivo culture evidence. Clin Cancer Res 21: 4420-4430, 2015.

5. Rajput P, Shukla SK and Kumar V: The HBx oncoprotein of hepatitis $\mathrm{B}$ virus potentiates cell transformation by inducing c-Myc-dependent expression of the RNA polymerase I transcription factor UBF. Virol J 12: 62, 2015.

6. Huang J, Wang Y, Guo Y and Sun S: Down-regulated microRNA-152 induces aberrant DNA methylation in hepatitis B virus-related hepatocellular carcinoma by targeting DNA methyltransferase 1. Hepatology 52: 60-70, 2010.

7. Yuan JH, Yang F, Chen BF, Lu Z, Huo XS, Zhou WP, Wang F and Sun SH: The histone deacetylase 4/SP1/microrna-200a regulatory network contributes to aberrant histone acetylation in hepatocellular carcinoma. Hepatology 54: 2025-2035, 2011.

8. Moyo B, Nicholson SA and Arbuthnot PB: The role of long non-coding RNAs in hepatitis B virus-related hepatocellular carcinoma. Virus Res 212: 103-113, 2016.

9. Nagano $\mathrm{T}$ and Fraser P: No-nonsense functions for long noncoding RNAs. Cell 145: 178-181, 2011.

10. Wilusz JE, Sunwoo H and Spector DL: Long noncoding RNAs: Functional surprises from the RNA world. Genes Dev 23: 1494-1504, 2009.

11. Mercer TR, Dinger ME and Mattick JS: Long non-coding RNAs: Insights into functions. Nat Rev Genet 10: 155-159, 2009.

12. Pandey RR, Mondal T, Mohammad F, Enroth S, Redrup L, Komorowski J, Nagano T, Mancini-Dinardo D and Kanduri C: Kcnqlot1 antisense noncoding RNA mediates lineage-specific transcriptional silencing through chromatin-level regulation. Mol Cell 32: 232-246, 2008.

13. Wang X, Arai S, Song X, Reichart D, Du K, Pascual G, Tempst P, Rosenfeld MG, Glass CK and Kurokawa R: Induced ncRNAs allosterically modify RNA-binding proteins in cis to inhibit transcription. Nature 454: 126-130, 2008.

14. Zhao Z, Li S, Song E and Liu S: The roles of ncRNAs and histone-modifiers in regulating breast cancer stem cells. Protein Cell 7: 89-99, 2016.
15. Ji P, Diederichs S, Wang W, Böing S, Metzger R, Schneider PM, Tidow N, Brandt B, Buerger H, Bulk E, et al: MALAT-1, a novel noncoding RNA, and thymosin beta4 predict metastasis and survival in early-stage non-small cell lung cancer. Oncogene 22: 8031-8041, 2003

16. Fu X, Ravindranath L, Tran N, Petrovics G and Srivastava S: Regulation of apoptosis by a prostate-specific and prostate cancer-associated noncoding gene, PCGEM1. DNA Cell Biol 25: 135-141, 2006.

17. Du Y, Kong G, You X, Zhang S, Zhang T, Gao Y, Ye L and Zhang $X$ : Elevation of highly up-regulated in liver cancer (HULC) by hepatitis B virus X protein promotes hepatoma cell proliferation via down-regulating p18. J Biol Chem 287: 26302-26311, 2012.

18. Yang F, Zhang L, Huo XS, Yuan JH, Xu D, Yuan SX, Zhu N, Zhou WP, Yang GS, Wang YZ, et al: Long noncoding RNA high expression in hepatocellular carcinoma facilitates tumor growth through enhancer of zeste homolog 2 in humans. Hepatology 54 : 1679-1689, 2011

19. Yuan JH, Yang F, Wang F, Ma JZ, Guo YJ, Tao QF, Liu F, Pan W, Wang TT, Zhou CC, et al: A long noncoding RNA activated by TGF- $\beta$ promotes the invasion-metastasis cascade in hepatocellular carcinoma. Cancer Cell 25: 666-681, 2014.

20. Ishibashi M, Kogo R, Shibata K, Sawada G, Takahashi Y, Kurashige J, Akiyoshi S, Sasaki S, Iwaya T, Sudo T, et al: Clinical significance of the expression of long non-coding RNA HOTAIR in primary hepatocellular carcinoma. Oncol Rep 29: 946-950, 2013.

21. Huang JL, Ren TY, Cao SW, Zheng SH, Hu XM, Hu YW, Lin L, Chen J, Zheng L and Wang Q: HBx-related long non-coding RNA DBH-AS1 promotes cell proliferation and survival by activating MAPK signaling in hepatocellular carcinoma. Oncotarget 6: 33791-33804, 2015.

22. Huang JF, Guo YJ, Zhao CX, Yuan SX, Wang Y, Tang GN, Zhou WP and Sun SH: Hepatitis B virus X protein (HBx)-related long noncoding RNA (lncRNA) down-regulated expression by $\mathrm{HBx}$ (Dreh) inhibits hepatocellular carcinoma metastasis by targeting the intermediate filament protein vimentin. Hepatology 57: 1882-1892, 2013.

23. Murata S, Mine T, Sugihara F, Yasui D, Yamaguchi H, Ueda T, Onozawa $S$ and Kumita $S$ : Interventional treatment for unresectable hepatocellular carcinoma. World J Gastroenterol 20: 13453-13465, 2014.

24. Margini C and Dufour JF: The story of HCC in NAFLD: From epidemiology, across pathogenesis, to prevention and treatment. Liver Int 36: 317-324, 2016.

25. Greten TF, Wang XW and Korangy F: Current concepts of immune based treatments for patients with HCC: From basic science to novel treatment approaches. Gut 64: 842-848, 2015.

26. Dufour JF, Bargellini I, De Maria N, De Simone P, Goulis I and Marinho RT: Intermediate hepatocellular carcinoma: Current treatments and future perspectives. Ann Oncol 24 (Suppl 2): ii24-ii29, 2013

27. Wu Y, Sarkissyan M and Vadgama JV: Epigenetics in breast and prostate cancer. Methods Mol Biol 1238: 425-466, 2015.

28. Kanwal R, Gupta K and Gupta S: Cancer epigenetics: An introduction. Methods Mol Biol 1238: 3-25, 2015.

29. Dawson MA and Kouzarides T: Cancer epigenetics: From mechanism to therapy. Cell 150: 12-27, 2012.

30. Yang N, Ekanem NR, Sakyi CA and Ray SD: Hepatocellular carcinoma and microRNA: New perspectives on therapeutics and diagnostics. Adv Drug Deliv Rev 81: 62-74, 2015.

31. Batista PJ and Chang HY: Long noncoding RNAs: Cellular address codes in development and disease. Cell 152: 1298-1307, 2013.

32. Lee JT: Epigenetic regulation by long noncoding RNAs. Science 338: 1435-1439, 2012.

33. Huarte M: The emerging role of lncRNAs in cancer. Nat Med 21: 1253-1261, 2015

34. Protzer U: Hepatitis: Epigenetic control of HBV by $\mathrm{HBx}$ protein-releasing the break? Nat Rev Gastroenterol Hepatol 12: 558-559, 2015.

35. Park IY, Sohn BH, Yu E, Suh DJ, Chung YH, Lee JH, Surzycki SJ and Lee YI: Aberrant epigenetic modifications in hepatocarcinogenesis induced by hepatitis B virus $\mathrm{X}$ protein. Gastroenterology 132: 1476-1494, 2007. 